\title{
A Search for Planetary Nebulae in M 33 and M 81
}

\author{
Laura Magrini, Mario Perinotto \\ Dipartimento di Astronomia e Scienza dello Spazio, Largo E. Fermi, 2 \\ 50125 Firenze, Italia
}

Romano L.M. Corradi

Isaac Newton Group of Telescopes, Apartado de Correos 321, 38700 Santa Cruz de La Palma, Canarias, Spain

Antonio Mampaso

Instituto de Astrofísica de Canarias, c. Vía Láctea $s / n$, 38200, La Laguna, Tenerife, Canarias, Spain

\begin{abstract}
We present a study of the PNe systems of two nearby galaxies: the spirals M 33 and M 81. The luminosity functions (PNLFs) for the two PNe systems were built and the distances to both galaxies were given $(0.84 \pm 0.09 \mathrm{Mpc}$ to $\mathrm{M} 33$ and $3.84 \pm 0.41 \mathrm{Mpc}$ to $\mathrm{M} 81)$. The behavior of $\mathrm{PNe}$ excitation was examined across both galaxies, finding no evidence for substantial differences in excitation between bulge and disk $\mathrm{PNe}$, nor for variations along the galaxian disks.
\end{abstract}

\section{Planetary Nebulae in $\mathrm{M} 33$ and $\mathrm{M} 81$}

Extragalactic Planetary Nebulae (PNe) are important targets in the study of the stellar populations of intermediate age in galaxies of different morphological types and in different chemical environments, and in the assessment of the kinematic properties of all morphological components of galaxies (disks, bulges, haloes). We show a study of the PNe systems of two nearby galaxies: the spiral galaxies M 33 and M 81 (Magrini et al. 2000, 2001; Magrini et al. 2001A). Large areas belonging to both galaxies and their external regions have been searched for emission-line objects, using the prime focus Wide Field Camera (WFC) of the $2.54 \mathrm{~m}$ Isaac Newton Telescope (La Palma, Spain). These objects have been identified with the usual on-band/off-band technique, i.e. by subtracting the galaxian stellar continuum from the $[\mathrm{O}$ III] and $\mathrm{H} \alpha+[\mathrm{N} \mathrm{II}]$ frames. The criteria adopted to identify PNe are the following ones: i) they should appear both in the $[\mathrm{O} \mathrm{III]}$ and $\mathrm{H} \alpha+[\mathrm{N} \mathrm{II}]$ frames but not in the continuum frame; ii) they should not be spatially resolved, i.e. they should have a stellar point spread function. At the distances of M $33(0.84 \pm 0.02 \mathrm{Mpc}$; Acker et al. 1991) and of M $81(3.50 \pm 0.40 \mathrm{Mpc}$, Jacoby et al. 1989) and with our seeing conditions, no $\mathrm{PN}$ can be resolved. A total of 131 candidate PNe, fulfilling the above presented criteria, have been identified in M 33 and 171 in M 81. A different field, centered in the intergalactic region between M 81 and M 82, allowed us to have the 
first detection of a stellar population, traced by $\mathrm{PNe}$, in the stream connecting M 81 and its companion M 82, which is being deeply investigated with new observations.

\section{The PNLFs and the behavior of PNe excitation}

The luminosity functions (PNLFs) for the two PNe systems were built and the distances to both galaxies were given fitting the PNLFs, within the completeness limit of the samples, to the "universal" PNLF (Jacoby 1989). We have obtained a distance of $0.84 \pm 0.09 \mathrm{Mpc}$ to $\mathrm{M} 33$ and of $3.84 \pm 0.41 \mathrm{Mpc}$ to $\mathrm{M} 81$. The behavior of PNe excitation, provided by the flux ratio $R=\frac{[O I I I]}{H \alpha+[N I I]}$, was examined across both galaxies (Magrini et al. 2001A), finding no evidence for substantial differences in excitation between bulge and disk $\mathrm{PNe}$, nor for variations along the galaxian disks. From the analysis of the catalogue of Galactic PNe by Acker et al. (1992), we found that the same result applies to the Milky Way, at variance with previous suggestions from Vorontsov-Vel'yaminov et al. (1975).

\section{Results from the study of Extragalactic Planetary Nebulae}

Future work is planned to study spectroscopically the individual candidates, confirm their nature and determine their physical properties. In fact, in spite of their importance, there are few spectroscopic data for extragalactic PNe and the abundances derived from their spectra have been calculated only for few Local Group galaxies. The distance to M 33 will allow us to use PNe spectra to derive chemical abundances of the brightest PNe and, from them, the existence of metallicity gradients throughout the galaxy. These data will also permit us to strengthen the validity of the use of the luminosity function of planetary nebulae to measure extragalactic distances, by quantifying the degree of contamination by other emission line objects (especially compact $\mathrm{H}$ II regions). At the distance of M 81 with presently available equipments PNe can not be used for chemical abundances determinations, but they are amongst the best suitable objects to study the kinematics of the various stellar populations, since they are created by stars with a wide range of masses.

\section{References}

Acker A., Ochsenbein F., Stenholm B., Tylenda R., Marcout J, Schon C., Freedman, W.L. 1991 ApJ, 372, 455

Magrini L., Corradi R.L.M., Mampaso A., Perinotto M. 2000, A\&A, 355, 713

Magrini L., Cardwell A., Corradi R.L.M., Mampaso A., Perinotto M. 2001, A\&A, 367, 498

Magrini L., Perinotto M., Corradi R.L.M., Mampaso A. 2001A, A\&A, 379, 90

Jacoby G. H., Ciardullo R., Holland C. F., Booth F. 1989 ApJ, 344, 704

Jacoby G. H. 1989 ApJ, 339, 39

Vorontsov-Vely'aminov B. A., Kostiakova E. B., Dokuchaeva O. D., Arkhipova V. P. 1975 AZh, 52, 264 